\title{
Comparative Study of the Metabolism of 1-Aminocyclopropane-1-carboxylic Acid and Senescence of Water-Stressed and ABA-Treated Excised Rice Leaves
}

\author{
Chien Teh Chen and Ching Huei Kao
}

\author{
Department of Agronomy, National Taiwan University, Taipei, Taiwan, Republic of China
}

\begin{abstract}
Changes in the metabolism of 1-aminocyclopropane-1-carboxylic acid (ACC) during senescence in the light in turgid, water-stressed, and ABA-treated; excised rice leaves were examined. The decreases in levels of $\mathrm{Chl}$ and protein were more rapid in the water-stressed and in the ABAtreated leaves than in the turgid leaves. In turgid leaves, levels of proline remained very low, but they increased considerably as a result of water stress or treatment with ABA. The production of ethylene was strongly inhibited by water stress and by ABA through the inhibition of the synthesis of ACC and/or the conversion of ACC to ethylene. In turgid leaves, the level of 1(malonylamino)cyclopropane-1-carboxylic acid (MACC) increased with time during incubation in the light. Water stress resulted in a pattern of accumulation of MACC similar to that in the turgid control. However, ABA blocked the malonylation of ACC.
\end{abstract}

Key words: Abscisic acid - Ethylene - Leaf senescence - Oryza sativa - Water stress.

Water stress has long been known to affect many physiological and developmental processes in plants (Bradford and Hsiao 1982, Hanson and Hitz 1982). Exogenous application of ABA also affects various physiological and developmental processes (Walton 1980). The levels of endogenous ABA rise markedly in response to water stress (Walton 1980). The increase is most dramatic in leaves, but it can also be observed in roots, stems, and xylem and phloem sap (Hoad 1975, 1978, Walton et al. 1976). Several reports indicate that ABA may play an important role in determining the pattern of plant development in the presence of water stress (Quarrie and Jones 1977, Watts et al. 1981).

Water stress has been shown to enhance the rate of senescence of intact plants and of detached organs (Brady et al. 1974, Dwivedi et al. 1979a, b, Gates 1968, Shah and Loomis 1965). In our early work with excised rice leaves we found that water stress accelerated senescence during the early stages of incubation in the dark, but it retarded senescence during later stages of incubation (Kao 1981). We do not know whether the effect of water stress on the senescence of excised rice leaves in the light is the same as that in darkness. The present investigation was

Abbreviations: ACC, 1-aminocyclopropane-1-carboxylic acid; MACC, 1-(malonylamino)cyclopropane-1-carboxylic acid. undertaken to examine whether exogenously applied ABA can mimic the effects of water stress on metabolic changes, in particular on the metabolism of $\mathrm{ACC}$ in excised rice leaves in the light.

\section{Materials and Methods}

Rice (Oryza sativa cv. Taichung Native 1) seedlings were cultured as previously described (Kao 1980b). The apical $3 \mathrm{~cm}$ of the third leaves of 12-day-old seedlings were used for experiments.

Water stress was applied by a previously described method (Kao 1981). Groups of twenty leaf segments were weighed and exposed to the vapor above a solution of $0.5 \mathrm{M}$ $\mathrm{NaCl}$. Water loss was determined by weighing the samples and expressed as a percentage of initial fresh weight. Similar segments of leaves were floated on $20 \mathrm{ml}$ of distilled water or a solution of $\mathrm{ABA}(0.1 \mathrm{mM})$ in a Petri dish to serve as turgid controls and $\mathrm{ABA}$-treated samples, respectively. All samples were kept at $27^{\circ} \mathrm{C}$ under light $\left(16.7 \mathrm{~W} \cdot \mathrm{m}^{-2}\right)$ provided by fluorescent lamps.

Chl, protein and proline were extracted and quantitated as described previously (Kao 1980a, 1981). For determination of amounts of ethylene liberated, leaf segments were placed vertically in test tubes which were closed with rubber stoppers. The ethylene in the gas phase of 
the enclosed tubes was determined by analysis of a $1-\mathrm{ml}$ sample withdrawn with a hypodermic syringe as described elsewhere (Kao and Yang 1983). ACC and MACC were extracted with $80 \%$ ethanol. After evaporation, an aliquot of the resulting aqueous extract was assayed for its ACC content by the method of Lizada and Yang (1979). For determination of MACC, another aliquot of the extract was passed over a column of cation-exchange resin, Dowex $50\left(\mathrm{H}^{+}\right.$form), to remove free amino acids, including ACC, and the effluent was hydrolyzed with $2 \mathrm{~N} \mathrm{HCl}$ at $100^{\circ} \mathrm{C}$ for $6 \mathrm{~h}$. The resulting $\mathrm{ACC}$ was then assayed. Levels of $\mathrm{ACC}$ and MACC are expressed as nmol per $g$ of initial fresh weight.

Levels of Chl, protein and proline contents are expressed as means with standard errors. Experiments on changes in rates of production of ethylene, and in levels of ACC and MACC were repeated three times, and identical trends were recorded. The data reported in Figure 4 are from a single experiment.

\section{Results}

Leaf width and fresh weight of leaf segments exposed to the vapor above a $0.5 \mathrm{M}$ solution of $\mathrm{NaCl}$, in the light, decreased rapidly for $24 \mathrm{~h}$ but subsequently remained unchanged (data not shown). The fresh weight at $24 \mathrm{~h}$ was $60 \%$ of the initial fresh weight. Leaf rolling was evident under our experimental conditions, indicating that the treatment did indeed cause water stress. In the case of leaf segments floated on water or a solution of $A B A(0.1 \mathrm{~mm})$,

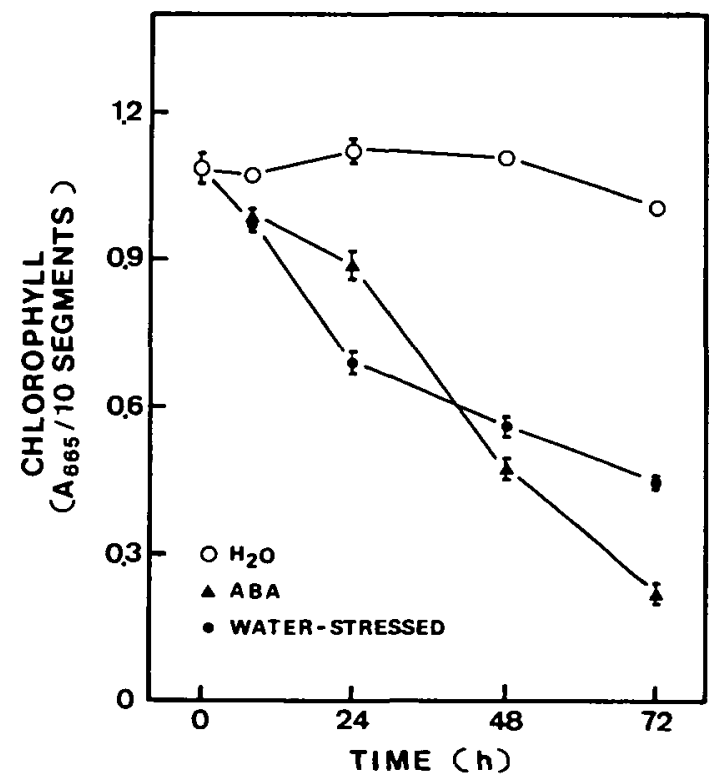

Fig. 1 Changes with time in Chl content of detached rice leaves. Turgid, water-stressed and ABA-treated leaves were examined. Values are averages with standard errors $(n=4)$. no leaf rolling was observed.

Figures 1 and 2 show the time course of changes in amounts of $\mathrm{Chl}$ and protein in leaf segments floated on water or on the solution of $\mathrm{ABA}$, or subjected to water stress in the light. In turgid leaf segments, the Chl content remained unchanged for the first $48 \mathrm{~h}$ of incubation in the light, but subsequently it decreased slightly. The $\mathrm{Chl}$ content in water-stressed or ABA-treated leaf segments decreased rapidly and significantly. The protein content of turgid leaf segments increased slightly after excision but subsequently remained unchanged. Levels of protein, like levels of Chl, fell considerably as a result of water stress or treatment with ABA.

Proline accumulates in leaves under water stress (Hanson and Hitz 1982). Proline also accumulates in response to the application of exogenous $\mathrm{ABA}$ in certain species (Aspinall 1980). Figure 3 shows the changes in levels of proline in excised rice leaves treated with ABA or subjected to water stress. In turgid leaves, the level of proline was very low and remained unchanged during incubation in the light. However, the level of proline was increased markedly as a result of water stress or treatment with ABA. The level of proline was much higher in excised, waterstressed leaves than in ABA-treated leaves.

Figure 4 shows that the production of ethylene in turgid leaves increased with increasing duration of incubation. The production of ethylene was inhibited by water stress or treatment with ABA. No increase in the production of ethylene in water-stressed or ABA-treated excised leaves was observed during a 24-h incubation. The rate of

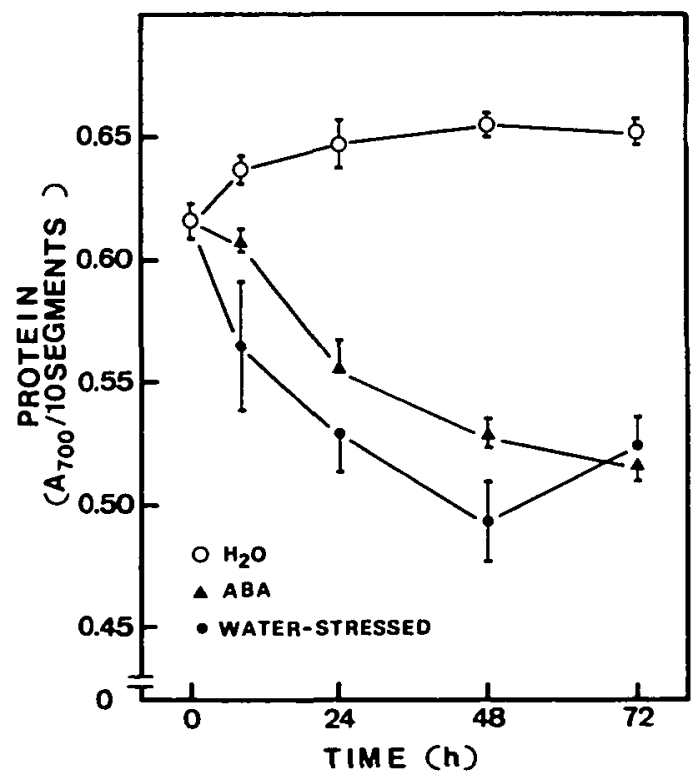

Fig. 2 Changes with time in protein content of detached rice leaves. Turgid, water-stressed and ABA-treated leaves were examined. Values are averages with standard errors $(n=4)$. 
production of ethylene is generally controlled by the level of ACC, the immediate precursor of ethylene (Yang and Hoffman 1984). ACC has been found to be converted into MACC in all tissues so far examined (Amrhein et al. 1982, Hoffman et al. 1982, 1983a). Malonylation of ACC can regulate the level of $\mathrm{ACC}$ which, in turn, might play a role in the control of the production of ethylene (Liu et al. 1985, Yang 1987). Thus, it would be of great interest to determine how water stress or ABA exerts its inhibitory effect on the production of ethylene. The effects of water stress and treatment with ABA on levels of ACC and MACC were examined and the results are shown in Figure 4. In turgid leaves, levels of $A C C$ increased during the first $8 \mathrm{~h}$ of incubation and decreased at $24 \mathrm{~h}$. Levels of MACC in turgid leaves increased progressively with time during the entire 24-h incubation. Water stress resulted in lower levels of ACC than those in turgid controls at 8 and $24 \mathrm{~h}$ after excision, although some increase was observed. These results suggest that a reduced rate of production of ethylene in water-stressed leaves is regulated by the inhibition of the synthesis of ACC. Since the difference in levels of ACC after a 24-h incubation between turgid controls and water-stressed samples was small but the difference in the amount of ethylene produced was large, the possibility that the conversion of ACC to ethylene is inhibited by water stress can not be ruled out. This possibility was tested by measuring ethylene production in the presence of a saturating concentration of ACC (10 mM). As indicated

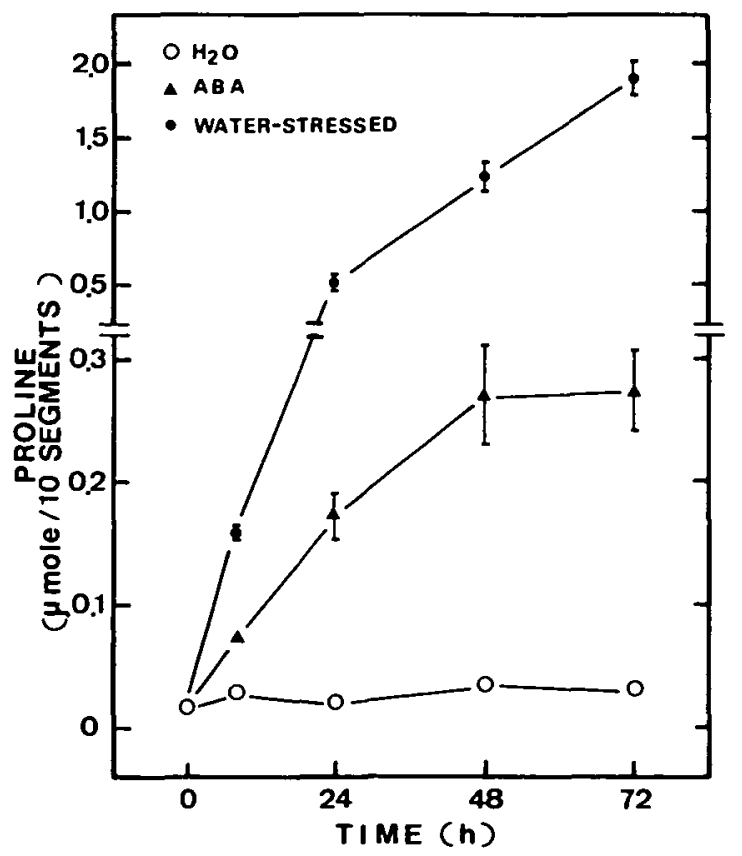

Fig. 3 Changes with time in proline content of detached rice leaves. Turgid, water-stressed and ABA-treated leaves were examined. Values are averages with standard errors $(n=4)$. in Figure 5, the conversion of ACC to ethylene was, indeed, inhibited by water stress at $24 \mathrm{~h}$, but not at 4 and $8 \mathrm{~h}$ after the start of the incubation.

Level of MACC in ABA-treated leaves remained low during the 24-h incubation, indicating that ABA blocked the conjugation of ACC (Fig. 4). Since neither conjugation of ACC nor increase in production of ethylene is observed in ABA-treated leaves, ACC would be expected to accumulate, if the synthesis of ACC is not impaired. However, this was not the case. Treatment with ABA resulted in lower levels of ACC than those in the turgid controls after 4- and 8-h incubations in the light, suggesting that the synthesis of $\mathrm{ACC}$ was inhibited by ABA. These results are consistent with those of McKeon et al. (1982). In addition to the reduced level of ACC in the ABA-treated leaves, the conversion of $A C C$ to ethylene was also inhibited by treatment with ABA throughout the entire duration of the in-

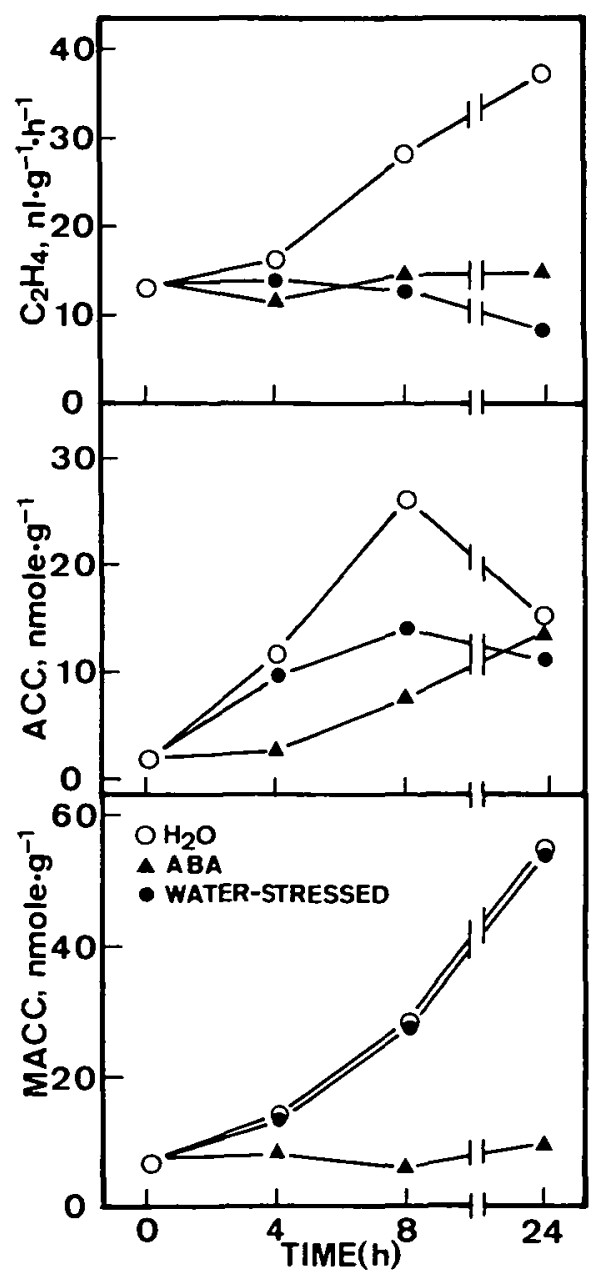

Fig. 4 Changes with time in rates of production of ethylene (upper), levels of ACC (middle) and levels of MACC (lower) in detached rice leaves. Turgid, water-stressed and ABA-treated leaves were examined. 


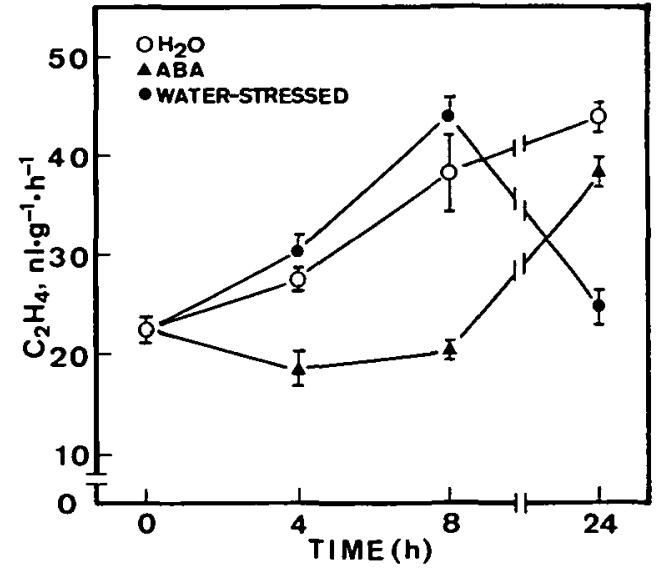

Fig. 5 Effect of water stress and of treatment with ABA on the conversion of $\mathrm{ACC}$ to ethylene. Leaf segments were pretreated with a saturating concentration of $A C C(10 \mathrm{~mm})$ for $2 \mathrm{~h}$. Control leaves were maintained turgid; experimental leaves were subjected to water stress or to treatment with ABA. Rates of production of ethylene were determined at the times indicated. Values are the average with standard errors $(n=5)$.

cubation (Fig. 5).

\section{Discussion}

Conventionally, the biochemical parameters used to indicate senescence of excised leaves are the decreases in the amounts of $\mathrm{Chl}$ and protein in the leaves (Dwivedi et al. 1979a, b, Kao and Yang 1983, Martin and Thimann 1972). Using these parameters, we demonstrated that senescence of excised rice leaves in the light is enhanced by water stress or treatment with ABA. These results are consistent with those reported by other investigators (Brady et al. 1974, Dwivedi et al. 1979b, Gates 1968, Shah and Loomis 1965). Our previous work, which involved incubation in the dark, showed that the decreases in amounts of $\mathrm{Chl}$ and protein were accelerated by water stress during the early stages of incubation but were retarded during the later stages (Kao 1981). The effect of water stress on senescence of excised rice leaves in the light appears different from that in the dark.

Previously, we suggested that the accumulation of proline in excised rice leaves after incubation in water might be a manifestation of metabolic changes associated with senescence (Wang et al. 1982). Our observation of the enhancement of the accumulation of proline by water stress in the light further supports the conclusion that water stress enhances senescence of excised rice leaves.

In excised, non-stressed wheat leaves, the rate of production of ethylene and levels of both ACC and MACC were very low throughout the entire 24 - or 22 -h incubation in the light (Apelbaum and Yang 1981, Hoffman et al. 1983b). However, the present investigation showed that the rate of production of ethylene and the levels of ACC and MACC in the light increased immediately and markedly after excision of rice leaves. In previous studies, we showed that the rate of production of ethylene in the light was lower than that in the dark (Kao and Yang 1982, 1983). We also demonstrated that inhibition of the production of ethylene by light during first $8 \mathrm{~h}$ of incubation was attributable to the inhibition in the conversion of ACC to ethylene (Kao and Yang 1982). Whereas, when the duration of the incubation time was longer than $8 \mathrm{~h}$, the reduced rate of production of ethylene in light-treated leaves was the result of reduced levels of ACC, due to the malonylation of ACC to MACC (Hurng et al. 1986).

Of particular interest is the finding that the production of ethylene is inhibited by water stress. This result is unexpected since the production of ethylene is generally considered to be enhanced by water stress (El-Betagy and Hall 1974, Hoffman et al. 1983b, Kimmerer and Kozlowski 1982, Neil et al. 1986, Wright 1977).

It has been shown that ABA exerts a marked inhibitory effect on the production of ethylene by oat and wheat leaves (Tan and Thimann 1989, Wright 1980) and on the auxin-induced production of ethylene in mung bean hypocotyls (Yoshii and Imaseki 1981). However, Riov and Yang (1987) recently demonstrated that ABA stimulated the auxin-induced production of ethylene in hypocotyls of light-grown mung bean. They also showed that ABA significantly increased the rate of production of ethylene in discs of tomato fruit and citrus leaf shortly after its application. Our results clearly show that the production of ethylene was significantly inhibited by ABA. The use of different species and different experimental conditions may be responsible for the discrepancies between the various results.

The present investigation demonstrated that the reduced production of ethylene in water-stressed and ABAtreated leaves is regulated by the inhibition of the synthesis of ACC and/or the conversion of ACC to ethylene. Several lines of evidence indicate that the conversion of ACC to ethylene in leaves is stimulated by carbon dioxide (Aharoni et al. 1979, Kao and Yang 1982, Preger and Gepstein 1984). Since ABA and water stress are known to close stomata (Radin and Ackerson 1982), the reduced rate of production of ethylene by ABA-treated and water-stressed leaves may be, at least in part, mediated by impairment of the inward passage of carbon dioxide.

With respect to the conversion of $\mathrm{ACC}$ to $\mathrm{MACC}$, our results revealed that (a) levels of MACC in non-stressed leaves increased significantly with increasing duration of incubation in the light, (b) there was no difference in levels of MACC between turgid and water-stressed leaves, and (c) ABA blocked the malonylation of ACC. These results are 
inconsistent with the results of Hoffman et al. (1983b) who found that levels of MACC in non-stressed, turgid leaves were low throughout the 22 -h incubation period, while in wilting leaves they increased markedly. It should be noted that in the present study the degree of water stress was $40 \%$ of water loss (based on initial fresh weight), whereas that of Hoffman et al. (1983b) was 9\%.

There is some experimental evidence of an increase in levels of ABA in detached leaves exposed to water stress. Thus, our work raises an important question. Why does ABA, but not water stress, block the malonylation of ACC? The distribution of ABA among the cellular compartments may differ between ABA-treated and waterstressed rice leaves or, alternatively, water stress may decrease levels of cytokinins (Hsiao 1973) with resultant suppression of the action of ABA on the formation of MACC. Elucidation of the mechanism of conjugation of ACC in ABA-treated and water-stressed rice leaves would be very useful in resolving these issues.

This research was supported financially by the National Science Council, Republic of China. The assistance of Dr. L. Y. $\mathrm{Su}$ is gratefully acknowledged.

\section{References}

Aharoni, A., Anderson, J. P. and Lieberman, M. (1978) Production and action of ethylene in sensecing leaf disks: effect of indole acetic acid, kinetin, silver ion and carbon dioxide. Plant Physiol. 64: 805-809.

Amrhein, N., Breuing, F., Eberle, J., Skorupka, H. and Tophof, S. (1982) The metabolism of 1-aminocyclopropane-1-carboxylic acid. In Plant Growth Substances 1982. Edited by Wareing, P. F. pp. 249-258. Academic Press, London.

Apelbaum, A. and Yang, S. F. (1981) Biosynthesis of stress ethylene induced by water deficit. Plant Physiol. 68: 594-596.

Aspinall, D. (1980) Role of abscisic acid and other hormones in adaptation to water stress. In Adaptation of Plants to Water and High-Temperature Stress. Edited by Turner, N. C. and Kramer, P. J. pp. 155-172. John Wiley \& Sons, New York.

Bradford, K. J. and Hsiao, T. C. (1982) Physiological responses to moderate water stress. In Encyclopedia of Plant Physiology, Vol. 12 B. Edited by Lange, O. L., Nobel, P. S., Osmond, C. B. and Ziegler, H. pp. 263-324. Springer-Verlag, Berlin.

Brady, C. J., Scott, N. S. and Munns, P. (1974) The interaction of water stress with the senescence pattern of leaves. In Mechanism of Regulation of Plant Growth. Edited by Bieleski, P. L., Ferguson, A. R. and Cresswell, M. M. pp. 403409. The Royal Society of New Zealand, Wellington.

Dwivedi, S., Kar, M. and Mishra, D. (1979a) Biochemical changes in excised leaves of Oryza sativa subjected to waterstress. Physiol. Plant. 45: 35-40.

Dwivedi, S., Kar, M. and Mishra, D. (1979b) Inorganic pyrophosphatase activity in water stressed excised rice leaves. Irriga- tion Sci. 1: 119-124.

El-Beltagy, A.S. and Hall, M. A. (1974) Effects of water stress upon endogenous ethylene levels in Vicia faba. New Phytol. 73: $47-60$.

Gates, C. J. (1968) Water deficits and growth of herbaceous plants. In Water Deficits and Plant Growth. Edited by Kozlowski, T. T. pp. 135-190. Academic Press, New York.

Hanson, A. D. and Hitz, W. D. (1982) Metabolic responses of mesophytes to plant water deficits. Annu. Rev. Plant Physiol. 33: 163-203.

Hoad, G. V. (1975) Effect of osmotic stress on abscisic acid levels in xylem sap of sunflower. Planta 124: 25-29.

Hoad, G. V. (1978) Effect of water stress on abscisic acid levels in white lupin (Lupinus albus L.) fruit, leaves and phloem exudate. Planta 142: 287-290.

Hoffman, N. E., Yang, S. F. and McKeon, T. (1982) Identification of 1-(malonylamino)cyclopropane-1-carboxylic acid as a major conjugate of 1-aminocyclopropane-1-carboxylic acid, an ethylene precursor in higher plants. Biochem. Biophys. Res. Commun. 104: 765-770.

Hoffman, N. E., Fu, J. R. and Yang, S. F. (1983a) Identification and metabolism of 1-(malonylamino)cyclopropane-1-carboxylic acid in germinating peanut seeds. Plant Physiol. 71: 197199.

Hoffman, N. E., Liu, Y. and Yang, S. F. (1983b) Changes in 1(malonylamino)cyclopropane-1-carboxylic acid content in wilted leaves in relation to their ethylene production rates and 1aminocyclopropane-1-carboxylic acid content. Planta 157: 518-523.

Hsiao, T. C. (1973) Plant responses to water stress. Annu. Rev. Plant Physiol. 24: 519-570.

Hurng, W. P., Su, L. Y. and Kao, C. H. (1986) Senescence of rice leaves XVI. Regulation by light. Bot. Bull. Academia Sinica 26: 163-174.

Kao, C. H. (1980a) Retardation of senescence by low temperature and benzyladenine in intact primary leaves of soybean. Plant Cell Physiol. 21: 339-344.

$\mathrm{KaO}, \mathrm{C} . \mathrm{H}$. (1980b) Senescence of rice leaves IV. Influence of benzyladenine on chlorophyll degradation. Plant Cell Physiol. 21: 1255-1262.

Kao, C. H. (1981) Senescence of rice leaves VI. Comparative study of the metabolic changes of senescing turgid and waterstressed excised leaves. Plant Cell Phhysiol. 22: 683-688.

Kao, C. H. and Yang, S. F. (1982) Light inhibition of the conversion of 1-aminocyclopropane-1-carboxylic acid to ethylene in leaves is mediated through carbon dioxide. Planta 155: 261266.

Kao, C. H. and Yang, S. F. (1983) Role of ethylene in the senescence of detached rice leaves. Plant Physiol. 73: 881-885.

Kimmerer, T. W. and Kozlowski, T. T. (1982) Ethylene, ethane, acetaldehyde and ethanol production by plants under stress. Plant Physiol. 69: 840-847.

Liu, Y., Hoffman, N. E. and Yang, S. F. (1985) Ethylene-promoted malonylation of 1-aminocyclopropane-1-carboxylic acid participates in autoinhibition of ethylene synthesis in grapefruit 
flavedo discs. Planta 164: 565-568.

Lizda, M. C. C. and Yang, S. F. (1979) A simple and sensitive assay for 1-aminocyclopropane-1-carboxylic acid. Anal. Biochem. 100: 140-145.

Martin, C. and Thimann, K. V. (1972) The role of protein synthesis in the senescence of leaves $I$. The formation of proteases. Plant Physiol. 49: 64-71.

McKeon, T. A., Hoffman, N. E. and Yang, S. F. (1982) Effect of plant pretreatments on ethylene production and synthesis of 1 aminocyclopropane-1-carboxylic acid in water-stressed wheat leaves. Planta 155: 437-443.

Neil, S. J., McGaw, B. A. and Horgan, R. (1986) Ethylene and 1-aminocyclopropane-1-carboxylic acid production in flacca, a wilty mutant of tomato, subjected to water deficiency and pretreatment with abscisic acid. J. Exp. Bot. 28: 193-203.

Preger, R. and Gepstein, S. (1984) Carbon dioxide-independent and -dependent components of light inhibition of the conversion of 1-aminocyclopropane-1-carboxylic acid to ethylene in oat leaves. Physiol. Plant. 60: 187-191.

Radin, J. W. and Ackerson, R. C. (1982) Does abscisic acid control stomatal closure during water stress? What's New in Plant Physiol. 12: 9-12.

Riov, J. and Yang, S. F. (1987) Effect of abscisic acid and methyl jasmonate on ethylene production. Plant Physiol. 83: S-175.

Shah, C. B. and Loomis, R. S. (1965) Ribonucleic acid and protein metabolism in sugar beet during drought. Physiol. Plant. 18: $240-254$.
Tan, Z.-Y. and Thimann, K. V. (1989) The roles of carbon dioxide and abscisic acid in the production of ethylene. Physiol. Plant. 75: 13-19.

Walton, D. C. (1980) Biochemistry and physiology of abscisic acid. Annu. Rev. Plant Physiol. 31: 453-489.

Walton, D. C., Harrison, M. A. and Cote, P. (1976) The effects of water stress on abscisic acid level and metabolism in roots of Phaseolus vulgaris L. and other plants. Planta 131: 141-144. Wang, C. Y., Cheng, S. H. and Kao, C. H. (1982) Senescence of rice leaves VII. Proline accumulation in senescing excised leaves. Plant Physiol. 69: 1348-1349.

Watts, S., Rodriguez, J. L., Evans, E. E. and Davies, W. J. (1981) Root and shoot growth of plants treated with abscisic acid. Ann. Bot. 47: 595-602.

Wright, S.T.C. (1977) The relationship between leaf water potential and the levels of abscisic acid and ethylene in excised wheat leaves. Planta 134: 183-189.

Wright, S. T. C. (1980) The effect of plant growth regulator treatments on the levels of ethylene emanating from excised turgid and wilted wheat leaves. Planta 148: 381-388.

Yang, S. F. (1987) Regulation of biosynthesis and action of ethylene. Acta Hortic. 201: 53-59.

Yoshii, H. and Imaseki, H. (1981) Biosynthesis of auxin-induced ethylene. Effect of indole-3-acetic acid, benzyladenine and abscisic acid on endogenous levels of 1-aminocyclopropane-1carboxylic acid (ACC) and ACC synthese. Plant Cell Physiol. 22: 369-379.

(Received July 27, 1989; Accepted March 12, 1990) 